Bull. Korean Math. Soc. 49 (2012), No. 1, pp. 197-204

http://dx.doi.org/10.4134/BKMS.2012.49.1.197

\title{
NORMAL FAMILIES AND SHARED HOLOMORPHIC FUNCTIONS
}

\author{
FENG LÜ
}

\begin{abstract}
In this paper, we study the problem of normal families and deduce some results, which improve and generalize several related theorems obtained by Pang [7], Fang and Xu [3], Lü, Xu, and Yi [6]. Meanwhile, some examples are given to show the sharpness of our results.
\end{abstract}

\section{Introduction and main results}

Let $f, g$ and $a$ be three holomorphic functions in a domain $D \subset \mathbb{C}$. Here, we denote the condition that $f(z)-a(z)=0$ implies $g(z)-a(z)=0$ by $f(z)=a(z) \Rightarrow g(z)=a(z)$. If $f(z)=a(z) \Rightarrow g(z)=a(z)$ and $g(z)=a(z) \Rightarrow$ $f(z)=a(z)$, we write $f(z)=a(z) \Leftrightarrow g(z)=a(z)$. In what follows, we assume that the reader is familiar with the basic notations and results in Nevanlinna value distribution theory (see, $[14,15]$ ).

One important subject in the theory of normal family is to find sufficient conditions for normality. According to Bloch's principle, a lot of normality criteria have been obtained by starting from Picard type theorems (see, $[1,2$, 4, 8, 9, 10]). The first attempt was made by Schwick [11] in 1992.

In a different way, Pang [7] and $\mathrm{Xu}$ [12] proved the following result.

Theorem A. Let $\mathcal{F}$ be a family of holomorphic functions in a domain $D$, and $a, b$ be distinct finite complex numbers. If $f(z)=a \Leftrightarrow f^{\prime}(z)=a$ and $f(z)=b \Leftrightarrow f^{\prime}(z)=b$ in $D$ for every $f \in \mathcal{F}$, then $\mathcal{F}$ is normal in $D$.

The following result was obtained by Fang and $\mathrm{Xu}$ [3] in 2002. They replaced the condition $f(z)=b \Leftrightarrow f^{\prime}(z)=b$ by $f(z)=b \Rightarrow f^{\prime}(z)=b$.

Theorem B. Let $\mathcal{F}$ be a family of holomorphic functions in a domain $D$, and $a, b$ be distinct finite complex numbers. If $f(z)=a \Leftrightarrow f^{\prime}(z)=a$ and $f(z)=b \Rightarrow f^{\prime}(z)=b$ in $D$ for every $f \in \mathcal{F}$, then $\mathcal{F}$ is normal in $D$.

Received October 7, 2010; Revised August 12, 2011.

2010 Mathematics Subject Classification. 30D45, 30D35.

Key words and phrases. holomorphic function, normal family, Nevanlinna theory, Cauchy theorem, linear differential polynomial.

The research was supported by the NSFC Tianyuan Mathematics Youth Fund (No.11026146) and the Fundamental Research Funds for the Central Universities (No.12CX04080A) and (No.10CX04038A). 
In 2009, Lü, Xu and Yi [6] improved Theorem B. They pointed out that Theorem B still holds if the condition $f(z)=a \Leftrightarrow f^{\prime}(z)=a$ is weakened to $f(z)=a \Rightarrow f^{\prime}(z)=a$.

Theorem C. Let $\mathcal{F}$ be a family of holomorphic functions in a domain D, let a and $b$ be two distinct complex numbers. If for all $f \in \mathcal{F}, f(z)=a \Rightarrow f^{\prime}(z)=a$ and $f(z)=b \Rightarrow f^{\prime}(z)=b$, then $\mathcal{F}$ is normal in $D$.

By studying the above theorems, we naturally ask what could happen if $f^{\prime}$ is replaced by a linear differential polynomial in $f$ with holomorphic coefficients?

In order to state our main results, we need the notation

$$
L[f]=a_{0} f^{\prime}+a_{1} f
$$

for a linear differential polynomial in $f$, where $a_{0}, a_{1}$ are holomorphic functions with $a_{0}(z) \neq 0$.

In the paper, by considering the above question, we obtain a result as follows, which is an improvement of the previous theorems.

Theorem 1.1. Let $\mathcal{F}$ be a family of holomorphic functions in a domain $D$, let $L[f]$ be defined as in (1.1), and let $a, b$ be two holomorphic functions in $D$. For each $f \in \mathcal{F}$, if

(1) $a \neq b$;

(2) $a-a_{1} a-a_{0} a^{\prime} \neq 0$

(3) $a-a_{1} a-a_{0} a^{\prime}$ and $b-a_{1} b-a_{0} b^{\prime}$ have no common zeros;

(4) $f(z)=a(z) \Rightarrow L[f](z)=a(z)$ and $f(z)=b(z) \Rightarrow L[f](z)=b(z)$, then $\mathcal{F}$ is normal in $D$.

Remark 1. Clearly, Theorem 1.1 is an improvement of the previous results. The following example shows that the condition (3) is necessary in Theorem 1.1 .

Example 1. Let $D=\{z:|z|<1\}$ and $k \geq 2$ be an integer, let $a(z)=z^{k}$ and $b(z)=2 z^{k}$, and let

$$
\mathcal{F}=\left\{f_{n}(z)=n z^{k}: n=3,4, \ldots ; z \in D\right\} .
$$

Suppose that $a_{0}=1$ and $a_{1}=0$. Then $L\left[f_{n}\right]=f_{n}^{\prime}$. For each $f_{n} \in \mathcal{F}$, we have that $f_{n}(z)=a(z) \Rightarrow L\left[f_{n}\right](z)=a(z)$ and $f_{n}(z)=b(z) \Rightarrow L\left[f_{n}\right](z)=b(z)$. Moreover,

$$
a(z)-a_{1}(z) a(z)-a_{0}(z) a^{\prime}(z)=a(z)-a^{\prime}(z)=z^{k-1}(z-k)
$$

and

$$
b(z)-a_{1}(z) b(z)-a_{0}(z) b^{\prime}(z)=b(z)-b^{\prime}(z)=2 z^{k-1}(z-k) .
$$

So $a-a_{1} a-a_{0} a^{\prime}$ and $b-a_{1} b-a_{0} b^{\prime}$ have a common zero $z=0$. Obviously, $\mathcal{F}$ is not normal in $D$.

Suppose that $a_{0}=1$ and $a_{1}=0$ in (1.1). Then the following corollary is an immediate consequence of Theorem 1.1. 
Corollary 1.2. Let $\mathcal{F}$ be a family of holomorphic functions in a domain $D$, and let $a, b$ be two holomorphic functions in $D$. For each $f \in \mathcal{F}$, if

(1) $a \neq b$ and $a-a^{\prime} \neq 0$;

(2) $a-a^{\prime}$ and $b-b^{\prime}$ have no common zeros;

(3) $f(z)=a(z) \Rightarrow f^{\prime}(z)=a(z)$ and $f(z)=b(z) \Rightarrow f^{\prime}(z)=b(z)$, then $\mathcal{F}$ is normal in $D$.

Remark 2. The following example shows that Corollary 1.2 is not valid for a family of meromorphic functions.

Example 2. Let $D=\{z:|z|<1\}$, let $a=1$ and $b=0$, and let

$$
\mathcal{F}=\left\{f_{n}(z)=\frac{(2 n z-1)^{2 n}}{(2 n z-1)^{2 n}-1}: n=1,2, \ldots ; z \in D\right\} .
$$

Clearly, for each $f_{n} \in \mathcal{F}$, we have that $f_{n}(z)=0 \Rightarrow f_{n}^{\prime}(z)=0, f_{n}(z) \neq 1$ and $a(z) \neq b(z)$. But $f_{n}^{\sharp}(0)=4 n^{2} \rightarrow \infty$ as $n \rightarrow \infty$. It follows from Marty criterion that $\mathcal{F}$ is not normal in $D$.

Remark 3. Recently, Xu and Qiu [13] derived a similar result to Theorem 1.1. The proof of our result has roots in their work and [5]. Some of the above examples can be found in [13].

\section{The lemma}

To prove our result, we need the well-known Zalcman lemma. For the proof of our result, Zalcman lemma is essential.

Zalcman Lemma ([16]). Let $\mathcal{F}$ be a family of functions holomorphic in a domain $D$. If $\mathcal{F}$ is not normal at $z_{0} \in D$, then there exist

(a) points $z_{n} \in D, z_{n} \rightarrow z_{0}$;

(b) functions $f_{n} \in \mathcal{F}$, and

(c) positive number $\rho_{n} \rightarrow 0$ such that $f_{n}\left(z_{n}+\rho_{n} \xi\right)=g_{n}(\xi) \rightarrow g(\xi)$ locally uniformly, where $g$ is a non-constant entire function.

\section{The proof of Theorem 1.1}

Since normality is a local property, it is sufficient to show that $\mathcal{F}$ is normal at $\forall z_{0} \in D$. We now distinguish between two cases.

Case 1. $a\left(z_{0}\right) \neq b\left(z_{0}\right)$ and $a-a_{1} a-\left.a_{0} a^{\prime}\right|_{z=z_{0}} \neq 0$.

Suppose, to the contrary, that $\mathcal{F}$ is not normal at $z_{0}$. By Zalcman lemma, there exist a sequence of functions $f_{n} \in \mathcal{F}$, a sequence of complex numbers $z_{n} \rightarrow z_{0}$ and a sequence of positive numbers $\rho_{n} \rightarrow 0$, such that

$$
g_{n}(\xi)=f_{n}\left(z_{n}+\rho_{n} \xi\right) \rightarrow g(\xi)
$$

converges locally uniformly in $\mathbb{C}$, where $g$ is a non-constant entire function. Noting that $\rho_{n} \rightarrow 0, z_{n} \rightarrow z_{0}$ and (3.1), we deduce that

$$
f_{n}\left(z_{n}+\rho_{n} \xi\right)-a\left(z_{n}+\rho_{n} \xi\right) \rightarrow g(\xi)-a\left(z_{0}\right)
$$


and

$$
f_{n}\left(z_{n}+\rho_{n} \xi\right)-b\left(z_{n}+\rho_{n} \xi\right) \rightarrow g(\xi)-b\left(z_{0}\right) .
$$

It follows from (3.1) that

$$
g_{n}^{\prime}(\xi)=\rho_{n} f_{n}^{\prime}\left(z_{n}+\rho_{n} \xi\right) \rightarrow g^{\prime}(\xi) .
$$

Combing (3.1), (3.4) and $a_{0}(z) \neq 0$ yields that

$$
\rho_{n} \frac{L\left[f_{n}\right]\left(z_{n}+\rho_{n} \xi\right)}{a_{0}\left(z_{n}+\rho_{n} \xi\right)}=\rho_{n} f_{n}^{\prime}\left(z_{n}+\rho_{n} \xi\right)+\rho_{n} \frac{a_{1}\left(z_{n}+\rho_{n} \xi\right) f_{n}\left(z_{n}+\rho_{n} \xi\right)}{a_{0}\left(z_{n}+\rho_{n} \xi\right)} \rightarrow g^{\prime}(\xi) .
$$

Next, we will prove that $g-a\left(z_{0}\right)$ and $g-b\left(z_{0}\right)$ have only multiple zeros.

Suppose that $g\left(\eta_{0}\right)-a\left(z_{0}\right)=0$. Noting that $g-a\left(z_{0}\right) \neq 0$, Hurwitz's theorem and (3.2), there exists a sequence $\eta_{n} \rightarrow \eta_{0}$ such that (for $n$ large enough)

$$
f_{n}\left(z_{n}+\rho_{n} \eta_{n}\right)=a\left(z_{n}+\rho_{n} \eta_{n}\right) .
$$

Then, the assumption $f(z)=a(z) \Rightarrow L[f](z)=a(z)$ leads to $L\left[f_{n}\right]\left(z_{n}+\right.$ $\left.\rho_{n} \eta_{n}\right)=a\left(z_{n}+\rho_{n} \eta_{n}\right)$. Furthermore, it follows from (3.5) that

$$
g^{\prime}\left(\eta_{0}\right)=\lim _{n \rightarrow \infty} \rho_{n} \frac{L\left[f_{n}\right]\left(z_{n}+a_{n} \eta_{n}\right)}{a_{0}\left(z_{n}+a_{n} \eta_{n}\right)}=\lim _{n \rightarrow \infty} \rho_{n} \frac{a\left(z_{n}+a_{n} \eta_{n}\right)}{a_{0}\left(z_{n}+a_{n} \eta_{n}\right)}=0,
$$

which implies that $g-a\left(z_{0}\right)$ has only multiple zeros. Similarly, we can derive that $g-b\left(z_{0}\right)$ has only multiple zeros.

We claim that $g(\xi) \neq a\left(z_{0}\right)$, which is proved as follows.

Suppose that $\xi_{0}$ is a zero of $g-a\left(z_{0}\right)$ with multiplicity $m$. Then $g^{(m)}\left(\xi_{0}\right) \neq 0$. Clearly, $m \geq 2$. So there exists a positive number $\delta_{1}$ such that

$$
g(\xi) \neq 0, g^{\prime}(\xi) \neq 0, g^{(m)}(\xi) \neq 0
$$

in $D_{\delta_{1}}^{o}=\left\{z: 0<\left|\xi-\xi_{0}\right|<\delta_{1}\right\}$.

Noting that $g \neq a\left(z_{0}\right)$, Rouché theorem and (3.2), there exist $\xi_{n, j}(j=$ $1,2, \ldots, m)$ on $D_{\delta_{1} / 2}=\left\{\xi:\left|\xi-\xi_{0}\right|<\delta_{1} / 2\right\}$ such that

$$
f_{n}\left(z_{n}+\rho_{n} \xi_{n, j}\right)=a\left(z_{n}+\rho_{n} \xi_{n, j}\right) .
$$

Then, we have

$$
L\left[f_{n}\right]\left(z_{n}+\rho_{n} \xi_{n, j}\right)=a\left(z_{n}+\rho_{n} \xi_{n, j}\right)(j=1,2, \ldots, m) .
$$

Let $A$ be defined as

$$
A=\frac{a-a_{1} a}{a_{0}} .
$$

Obviously, $A$ is holomorphic in $D$. Combining (3.7), (3.8) and the form of $L\left[f_{n}\right]$ yields

$$
f_{n}^{\prime}\left(z_{n}+\rho_{n} \xi_{n, j}\right)=A\left(z_{n}+\rho_{n} \xi_{n, j}\right)(j=1,2, \ldots, m)
$$

Set

$$
G_{n}(\xi)=f_{n}\left(z_{n}+\rho_{n} \xi\right)-a\left(z_{n}+\rho_{n} \xi\right) .
$$

Then $G_{n}\left(\xi_{n, j}\right)=0(j=1,2, \ldots, m)$. 
Observing that $a-a_{1} a-\left.a_{0} a^{\prime}\right|_{z=z_{0}} \neq 0$, we obtain (for $n$ large enough)

$$
a-a_{1} a-\left.a_{0} a^{\prime}\right|_{z=z_{n}+\rho_{n} \xi_{n, j}} \neq 0 .
$$

Furthermore, we deduce that (for $n$ large enough)

$$
\begin{aligned}
G_{n}^{\prime}\left(\xi_{n, j}\right) & =\rho_{n}\left(f_{n}^{\prime}\left(z_{n}+\rho_{n} \xi_{n, j}\right)-a^{\prime}\left(z_{n}+\rho_{n} \xi_{n, j}\right)\right) \\
& =\rho_{n}\left(A\left(z_{n}+\rho_{n} \xi_{n, j}\right)-a^{\prime}\left(z_{n}+\rho_{n} \xi_{n, j}\right)\right) \\
& =\left.\rho_{n} \frac{a-a_{1} a-a_{0} a^{\prime}}{a_{0}}\right|_{z=z_{n}+\rho_{n} \xi_{n, j}} \neq 0,
\end{aligned}
$$

which implies that each $\xi_{n, j}$ is a simple zero of $G_{n}$. That is $\xi_{n, j} \neq \xi_{n, i}(1 \leq$ $i \neq j \leq m)$.

Set

$$
K_{n}(\xi)=\rho_{n} \frac{L\left[f_{n}\right]\left(z_{n}+\rho_{n} \xi\right)-a\left(z_{n}+\rho_{n} \xi\right)}{a_{0}\left(z_{n}+\rho_{n} \xi\right)}
$$

Then

$$
K_{n}(\xi) \rightarrow g^{\prime}(\xi)
$$

and $K_{n}\left(\xi_{n, j}\right)=0(j=1,2, \ldots, m)$. From (3.6), we have

$$
\lim _{n \rightarrow \infty} \xi_{n, j}=\xi_{0}(j=1,2, \ldots, m) .
$$

By (3.6), (3.10) and the fact that $K_{n}(\xi)$ has $m$ zeros $\xi_{n, j}(j=1,2, \ldots, m)$ in $D_{\delta_{1} / 2}, \xi_{0}$ is a zero of $g^{\prime}$ with multiplicity $m$, and thus $g^{(m)}\left(\xi_{0}\right)=0$. This is a contradiction and hence, the claim is proved.

By Nevanlinnas first and second fundamental theorems, we derive that

$$
\begin{aligned}
T(r, g) & \leq \bar{N}\left(r, \frac{1}{g-a\left(z_{0}\right)}\right)+\bar{N}\left(r, \frac{1}{g-b\left(z_{0}\right)}\right)+S(r, g) \\
& \leq \frac{1}{2} N\left(r, \frac{1}{g-b\left(z_{0}\right)}\right)+S(r, g) \leq \frac{1}{2} T(r, g)+S(r, g),
\end{aligned}
$$

which indicates that $T(r, g)=S(r, g)$, a contradiction. Thus, $\mathcal{F}$ is normal at $z_{0}$ and the proof of Case 1 is finished.

Case 2. $a\left(z_{0}\right)=b\left(z_{0}\right)$ or $a-a_{1} a-\left.a_{0} a^{\prime}\right|_{z=z_{0}}=0$.

Since $a \neq b$ and $a-a_{1} a-a_{0} a^{\prime} \neq 0$, then there exists $r>0$ such that $a(z) \neq b(z)$ and $a(z)-a_{1}(z) a(z)-a_{0}(z) a^{\prime}(z) \neq 0$ in $D^{\prime}\left(z_{0}, r\right)=\{z: 0<$ $\left.\left|z-z_{0}\right|<r\right\} \subset D$.

It follows from Case 1 that $\mathcal{F}$ is normal in $D^{\prime}\left(z_{0}, r\right)$. Then for any sequence $\left\{f_{n}\right\} \subset \mathcal{F}$, there exists a subsequence $\left\{f_{n, j}\right\}$ such that $\left\{f_{n, j}\right\}$ converges locally uniformly to a function $h$ in $D^{\prime}\left(z_{0}, r\right)$, where $h$ is either holomorphic or identically infinite in $D^{\prime}\left(z_{0}, r\right)$.

In the following, we consider two subcases.

Subcase 2.1. $h$ is holomorphic in $D^{\prime}\left(z_{0}, r\right)$.

Then, there exists a positive number $M$ such that $|h(z)| \leq M$ in $\left|z-z_{0}\right|=$ $r / 2$. It follows that $\left|f_{n, j}(z)\right| \leq 2 M$ on $\left|z-z_{0}\right|=r / 2$ for large $j$. By the 
maximum principle, we have $\left|f_{n, j}(z)\right| \leq 2 M$ in $D\left(z_{0}, r / 2\right)=\left\{z:\left|z-z_{0}\right| \leq\right.$ $r / 2\}$. Then $h$ is bounded in $D\left(z_{0}, r / 2\right)$, and $h$ extends to be holomorphic in $D\left(z_{0}, r / 2\right)$. Again by the maximum principle, we have $f_{n, j}(z) \rightarrow h(z)$ in $D\left(z_{0}, r / 2\right)$.

Subcase 2.2. $h=\infty$.

We consider again two subcases.

Subcase 2.2.1. $a-a_{1} a-\left.a_{0} a^{\prime}\right|_{z=z_{0}}=0$.

Since $a-a_{1} a-a_{0} a^{\prime}$ and $b-a_{1} b-a_{0} b^{\prime}$ have no common zeros, then $b-a_{1} b-$ $\left.a_{0} b^{\prime}\right|_{z=z_{0}} \neq 0$. So, there exists a positive number $r^{\prime}<r$ such that

$$
b(z)-a_{1}(z) b(z)-a_{0}(z) b^{\prime}(z) \neq 0
$$

in $D\left(z_{0}, r^{\prime}\right)=\left\{z:\left|z-z_{0}\right|<r^{\prime}\right\} \subset D$. Suppose that $z_{n}$ is a zero of $f_{n, j}-b$ in $D\left(z_{0}, r^{\prime}\right)$. Then, we have $f_{n, j}\left(z_{n}\right)=b\left(z_{n}\right)$ and $L\left[f_{n, j}\right]\left(z_{n}\right)=b\left(z_{n}\right)$. In view of $L[f]=a_{0} f^{\prime}+a_{1} f$, we deduce

$$
f_{n, j}^{\prime}\left(z_{n}\right)=\left.\frac{b-a_{1} b}{a_{0}}\right|_{z=z_{n}} .
$$

Let $H_{n, j}=f_{n, j}-b$. Then $H_{n, j}\left(z_{n}\right)=0$ and

$$
H_{n, j}^{\prime}\left(z_{n}\right)=f_{n, j}^{\prime}\left(z_{n}\right)-b^{\prime}\left(z_{n}\right)=\left.\frac{b-a_{1} b-a_{0} b^{\prime}}{a_{0}}\right|_{z=z_{n}} \neq 0,
$$

which implies that $f_{n, j}-b$ just has simple zeros in $D\left(z_{0}, r^{\prime}\right)$.

So the function $\frac{L\left[f_{n, j}\right]-b}{f_{n, j}-b}$ is holomorphic in $D\left(z_{0}, r^{\prime}\right)$. Let $0<r_{1}<r^{\prime}$ and $\Gamma:=\left\{z:\left|z-z_{0}\right|=r_{1}\right\}$. By Cauchy theorem we conclude that

$$
\int_{\Gamma} \frac{L\left[f_{n, j}\right](z)-b(z)}{f_{n, j}(z)-b(z)} d z=0
$$

Noting that $f_{n, j}-b \rightarrow \infty$ on $\Gamma$, we derive that (for sufficiently large $n$ )

$$
\left|\int_{\Gamma} \frac{a_{1}(z) b(z)+a_{0}(z) b^{\prime}(z)-b(z)}{f_{n, j}(z)-b(z)} d z\right| \leq \pi
$$

By $n\left(\Gamma, \frac{1}{f_{n, j}-b}\right)$ we denote the number of zeros of $f_{n, j}-b$ in $D\left(z_{0}, r\right)=\{z$ : $\left.\left|z-z_{0}\right|<r_{1}\right\}$. From the argument principle, (3.13) and (3.14) (for sufficiently large $n$ ), we obtain that

$$
\begin{aligned}
& n\left(\Gamma, \frac{1}{f_{n, j}-b}\right) \\
= & \left|\frac{1}{2 \pi i} \int_{\Gamma} \frac{f_{n, j}^{\prime}(z)-b^{\prime}(z)}{f_{n, j}(z)-b(z)} d z\right| \\
= & \left|\frac{1}{2 \pi i} \int_{\Gamma} \frac{a_{0}(z) f_{n, j}^{\prime}(z)-a_{0}(z) b^{\prime}(z)}{a_{0}(z)\left[f_{n, j}(z)-b(z)\right]} d z\right| \\
= & \left|\frac{1}{2 \pi i} \int_{\Gamma} \frac{L\left[f_{n, j}\right](z)-b(z)-a_{1}(z) f_{n, j}(z)+b(z)-a_{0}(z) b^{\prime}(z)}{a_{0}(z)\left[f_{n, j}(z)-b(z)\right]} d z\right|
\end{aligned}
$$




$$
\begin{aligned}
\leq & \left|\frac{1}{2 \pi i} \int_{\Gamma} \frac{L\left[f_{n, j}\right](z)-b(z)}{a_{0}(z)\left[f_{n, j}(z)-b(z)\right]} d z\right|+\left|\frac{1}{2 \pi i} \int_{\Gamma} \frac{a_{1}(z)\left[f_{n, j}(z)-b(z)\right]}{a_{0}(z)\left[f_{n, j}(z)-b(z)\right]} d z\right| \\
& +\left|\frac{1}{2 \pi i} \int_{\Gamma} \frac{a_{1}(z) b(z)+a_{0}(z) b^{\prime}(z)-b(z)}{a_{0}(z)\left[f_{n, j}(z)-b(z)\right]} d z\right| \leq \frac{1}{2},
\end{aligned}
$$

which implies that

$$
n\left(\Gamma, \frac{1}{f_{n, j}-b}\right)=0 .
$$

So $f_{n, j}-b$ has no zeros in $D\left(z_{0}, r_{1}\right)$. Thus, $\frac{1}{f_{n, j}-b}$ is holomorphic and $\frac{1}{f_{n, j}-b} \rightarrow$ 0 on $D^{\prime}\left(z_{0}, r_{1}\right)$. Similarly as in Case 2.1 , we can deduce $f_{n, j} \rightarrow \infty$ in $D\left(z_{0}, r_{1}\right)$.

Subcase 2.2.2. $a-a_{1} a-\left.a_{0} a^{\prime}\right|_{z=z_{0}} \neq 0$.

Then, there exists a positive number $r^{\prime \prime}<r$ such that

$$
a(z)-a_{1}(z) a(z)-a_{0}(z) a^{\prime}(z) \neq 0
$$

in $D\left(z_{0}, r^{\prime \prime}\right)=\left\{z:\left|z-z_{0}\right|<r^{\prime \prime}\right\} \subset D$. Furthermore, in a similar way as in Subcase 2.2.1, it is easy to deduce that $f_{n, j}(z) \rightarrow \infty$ in $D\left(z_{0}, r^{\prime \prime}\right)$.

Thus, the proof of Case 2 is finished. Combining Case 1 and 2 yields that $\mathcal{F}$ is normal at $z_{0}$, which completes the proof of Theorem 1.1.

\section{References}

[1] H. H. Chen and M. L. Fang, Shared values and normal families of meromorphic functions, J. Math. Anal. Appl. 260 (2001), no. 1, 124-132.

[2] H. H. Chen and X. H. Hua, Normal families concerning shared values, Israel J. Math. 115 (2000), 355-362.

[3] M. L. Fang and Y. Xu, Normal families of holomorphic functions and shared values, Israel J. Math. 129 (2002), 125-141.

[4] M. L. Fang and L. Zalcman, Normal families and shared values of meromorphic functions, Ann. Polon. Math. 80 (2003), 133-141.

[5] J. Grahl and C. Meng, Entire functions sharing a polynomial with their derivatives and normal families, Analysis (Munich) 28 (2008), no. 1, 51-61.

[6] F. Lü, J. F. Xu, and H. X. Yi, Uniqueness theorems and normal families of entire functions and their derivatives, Ann. Polon. Math. 95 (2009), no. 1, 67-75.

[7] X. C. Pang, Shared values and normal families, Analysis (Munich) 22 (2002), no. 2, $175-182$.

[8] X. C. Pang and L. Zalcman, Normality and shared values, Ark. Mat. 38 (2000), no. 1, $171-282$.

[9] _ Normal families and shared values, Bull. London Math. Soc. 32 (2000), no. 3, $325-331$

[10] J. Schiff, Normal Families, Springer-Verlag, New York/Berlin, 1993.

[11] W. Schwick, Sharing values and normality, Arch. Math. (Basel) 59 (1992), no. 1, 50-54.

[12] Y. Xu, Normality criteria concerning sharing values, Indian J. Pure Appl. Math. 30 (1999), no. 3, 287-293.

[13] Y. Xu and H. L. Qiu, An improvement of Montel's criterion, J. Math. Anal. Appl. 343 (2008), no. 2, 1075-1079.

[14] L. Yang, Value Distribution Theory, Springer-Verlag \& Science Press, Berlin, 1993.

[15] C. C. Yang and H. X. Yi, Uniqueness Theory of Meromorphic Functions, Science Press, Beijing, 2003. 
[16] L. Zalcman, Normal families: New perspectives, Bull. Amer. Math. Soc. (N.S.) 35 (1998), no. 3, 215-230.

Department of Mathematics

China University of Petroleum

Dongying, Shandong, 257061, P. R. China

E-mail address: 1vfeng18@gmail.com 